\title{
The novel and critical role of von Hippel-Lindau in nephron formation
}

\author{
Soyoung Park, So-mi Kang, Bum-Joon Park \\ Department of Molecular Biology, Pusan National University, Busan, Republic of Korea \\ Correspondence to: Bum-Joon Park. Pusan National University, 2, Busandaehak-ro, 63beon-gil, Geumjeong-gu, Busan 46241, Republic of Korea. \\ Email: bjpark1219@pusan.ac.kr. \\ Provenance: This is an invited article commissioned by the Section Editor Dr. Xiao Li (Department of Urology, Jiangsu Cancer Hospital, Jiangsu \\ Institute of Cancer Research, Nanjing Medical University Affiliated Cancer Hospital, Nanjing, China). \\ Comment on: Cargill K, Hemker SL, Clugston A, et al. Von Hippel-Lindau Acts as a Metabolic Switch Controlling Nephron Progenitor \\ Differentiation. J Am Soc Nephrol 2019;30:1192-205.
}

Submitted Sep 11, 2019. Accepted for publication Sep 29, 2019.

doi: 10.21037/atm.2019.09.159

View this article at: http://dx.doi.org/10.21037/atm.2019.09.159

Loss of von Hippel-Lindau gene (VHL) causes rare genetic disorder, characterized by clear cell renal cell carcinoma (ccRCC), pheochromocytomas, and hemangioblastomas (blood vessel tumors) (1-5). Well confirmed role of VHL is an E3 ligase for hypoxia-induced factors (HIFs) under normaxia condition. Under the presence of Oxygen, proline-hydroxylase, an iron containing dioxygenase, makes hydroxyproline of HIF-1, which provides recognition residue of VHL. VHL-mediated poly-ubiquitylated HIF-1 is degraded by proteasome. However, when oxygen concentration is dropped down (so called hypoxia condition), HIF- $1 \alpha$ can escape the VHL-mediated proteasomal degradation and serve as transcription factor with HIF-1 $\beta$ and induces many kinds of hypoxia-related genes such as vascular endothelial cell growth factor (VEGF) for neo-vascularization, glucose transporter-1 (Glut1) for promoting the glycose uptake and etc. $(6,7)$.

Thus, tumor suppressive role of VHL in hemangioblastoma is easily understood, considering the working mechanism of VHL. VHL would be sensor for oxygen and regulate endothelial cell growth and differentiation as well as epithelial cell proliferation through nutrients supplement and uptake. However, the role of VHL in other kinds of cancers, in particular, renal cell carcinoma (RCC) has not been clearly demonstrated until now, despite $70 \%$ of sporadic RCC show deficiency of VHL.

Considering the facts that kidney is well vascularized organ and the origin of RCC is not endothelial cell, HIF-1 mediated neo-vascularization seems not to be essential reason for VHL deletion in RCC. In addition, physiological role of VHL during embryonic development is covered due to early embryonic lethality of VHL knock out mouse model (8). Since $\mathrm{VHL}^{-/-}$mice are died at E10.5 to E12.5 days due to failure of extraembryonic vascular development, intracellular function during kidney development has not been clearly described.

In recent, Cargill et al., suggest very interesting function of VHL in kidney development, in particular, Nephron formation (9). They focused the role of oxygen concentration during kidney formation. In fact, hypoxia induces nephron deficit $(10,11)$ and oxygen level is changed from hypoxia to normoxia during kidney development (12). To avoid the embryonic lethality of VHL deficiency, they used conditional knock out mouse models and focused on differentiation of Nephron progenitor cells. They generate Six2-Cre; $\mathrm{VHL}^{\mathrm{f} / \mathrm{f}}$ mouse model for Six $2+$ nephron progenitor cell specific VHL deletion $\left(V H L^{N P_{-1}}\right)$. Using these mouse model, they showed that VHL is dispensable of early kidney development (until E13.5), because kidney is still hypoxic condition. However, from E15.5 (at this stage, oxygen supplement is increased), VHL deficient nephron progenitor cells showed the defect on mature nephron formation (glomeruli). In fact, the number of matured nephron is dramatically decreased in VHL deficient mice. Finally, $V H L^{N P-/-}$ mice died around P30. Cargill et al., also prove that VHL is dispensable for renal vesicle differentiation and function through another conditional knock out mouse model (Wnt4-cre; VHLf/f, $V H L^{R V-/}$ ). 
Indeed, these mice did not show significant differentiation in body weight, Kidney weight and kidney histology (9).

The authors also show that reduction of Nephron is resulted from defect of differentiation of nephron progenitor cells through expression analysis. Furthermore, they revealed that glycolysis and mitochondrial respiration rate are mainly altered by VHL (9). In fact, deficiency of VHL reduced the mitochondrial respiration and increased glycolysis. These results implied that physiological role of VHL-HIF1 axis is not only oxygen sensor but also molecular switch of mitochondrial respiration in nephron progenitor cell. Based on these results, we assume that loss of VHL may induce glycolysis without mitochondrial respiration (Warburg effect) as well as increase nephron progenitor cell population, which would contribute to tumor occurrence.

These results suggest that VHL is a critical regulator of energy metabolism as well as nephron progenitor cell differentiation. As author's discussion, increase of glycolysis and decrease of mitochondrial respiration (Warburg effect) is well known trait of cancer cell. Although author did not provide the direct evidence about relevance of cancer, it would be very useful for the role of VHL in human cancer, particularly RCC. If loss of VHL can trigger the Warburg effect in RCC and suppress cell differentiation, in my thought, it would be the tumor suppressive role of VHL in RCC, what we have looking for.

\section{Acknowledgments}

Funding: This work was supported by a 2-Year Research Grant from Pusan National University (2019-2021).

\section{Footnote}

Conflicts of Interest: The authors have no conflicts of interest to declare.

Ethical Statement: The authors are accountable for all aspects of the work in ensuring that questions related to the accuracy or integrity of any part of the work are appropriately investigated and resolved.

\section{References}

1. Cancer Genome Atlas Research Network. Comprehensive molecular characterization of clear cell renal cell carcinoma. Nature 2013;499:43-9.

2. Brauch $\mathrm{H}$, Weirich $\mathrm{G}$, Brieger J, et al. VHL alterations in human clear cell renal cell carcinoma: Association with advanced tumor stage and a novel hot spot mutation. Cancer Res 2000;60:1942-8.

3. Dahia PL. Pheochromocytoma and paraganglioma pathogenesis: Learning from genetic heterogeneity. Nature Reviews Cancer 2014;14:108-19.

4. Burnichon N, Vescovo L, Amar L, et al. Integrative genomic analysis reveals somatic mutations in pheochromocytoma and paraganglioma. Hum Mol Genet 2011;20:3974-85.

5. Gläsker S, Bender BU, Apel TW, et al. Reconsideration of biallelic inactivation of the VHL tumour suppressor gene in hemangioblastomas of the central nervous system. J Neurol Neurosurg Psychiatry 2001;70:644-8.

6. Bernhardt WM, Schmitt R, Rosenberger C, et al. Expression of hypoxia-inducible transcription factors in developing human and rat kidneys. Kidney Int 2006;69:114-22.

7. Iyer NV, Kotch LE, Agani F, et al. Cellular and developmental control of $\mathrm{O} 2$ homeostasis by hypoxiainducible factor $1 \alpha$. Genes Dev 1998;12:149-62.

8. Gnarra JR, Ward JM, Porter FD, et al. Defective placental vasculogenesis causes embryonic lethality in VHLdeficient mice. Proc Natl Acad Sci U S A 1997;94:9102-7.

9. Cargill K, Hemker SL, Clugston A, et al. Von hippellindau acts as a metabolic switch controlling nephron progenitor differentiation. J Am Soc Nephrol 2019;30:1192-205.

10. Rymer C, Paredes J, Halt K, et al. Renal blood flow and oxygenation drive nephron progenitor differentiation. Am J Physiol Renal Physiol 2014;307:F337-45.

11. Gonzalez-Rodriguez P Jr, Tong W, Xue Q, et al. Fetal hypoxia results in programming of aberrant angiotensin ii receptor expression patterns and kidney development. Int J Med Sci 2013;10:532-8.

12. Hemker SL, Sims-Lucas S, Ho J. Role of hypoxia during nephrogenesis. Pediatr Nephrol 2016;31:1571-7.

Cite this article as: Park S, Kang SM, Park BJ. The novel and critical role of von Hippel-Lindau in nephron formation. Ann Transl Med 2019;7(Suppl 8):S319. doi: 10.21037/ atm.2019.09.159 